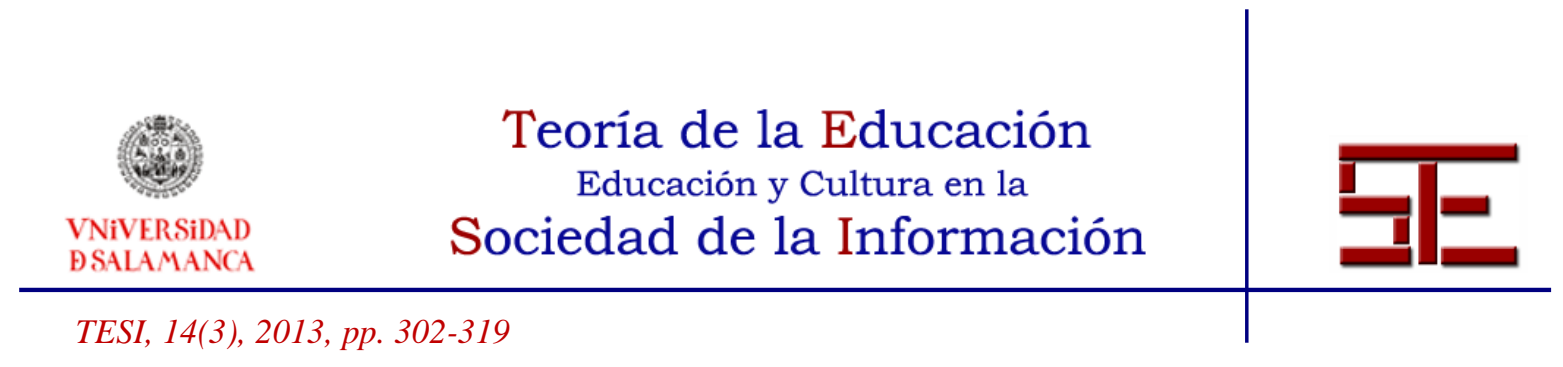

\title{
EXPLORANDO EL POTENCIAL EDUCATIVO DE LOS ENTORNOS VIR- TUALES 3D
}

Resumen: Los entornos virtuales 3D son sistemas tecnológicos avanzados, con múltiples potencialidades en el proceso de enseñanza-aprendizaje.

En los últimos años diferentes instituciones han impulsado la necesaria adquisición de una serie de competencias clave para el siglo XXI tales como la iniciativa, el trabajo en equipo, la creatividad, la flexibilidad o la competencia digital.

Los entornos virtuales multiusuario o espacios de simulación 3D son sistemas inmersivos, interactivos, personalizables, accesibles y programables, que permiten diseñar actividades complejas de carácter educativo dando respuesta a estas competencias clave. Para ello resulta necesario establecer una adecuada estrategia didáctica que permita poner en acción conocimientos y habilidades, y diseñar adecuados mecanismos de registro y sistematización.

En el presente artículo se exponen las potencialidades de estos entornos y se presentan dos experiencias realizadas en estos espacios 3D: (1) para el desarrollo de la competencia de trabajo en equipo y autogestión, y (2) para la evaluación de la competencia digital en futuros maestros.

Palabras clave: Entornos virtuales 3D; Mundos virtuales; competencias clave; competencia digital; trabajo en equipo; autogestión.

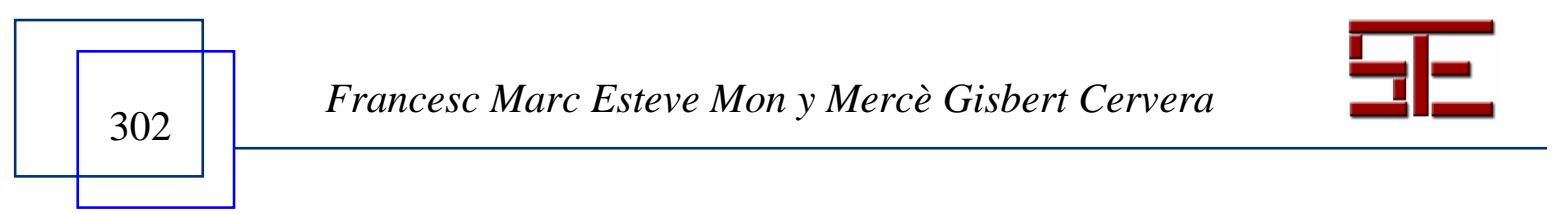




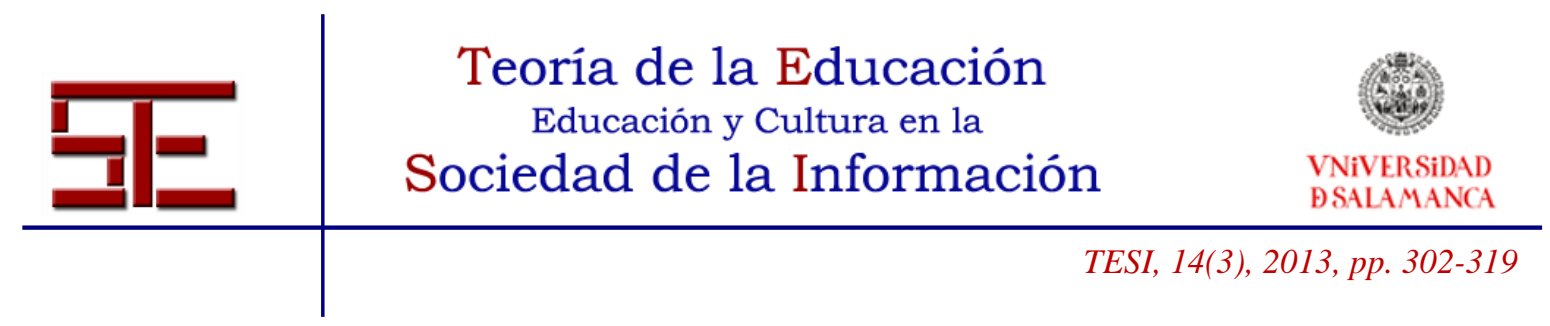

\section{EXPLORING THE EDUCATIONAL POTENTIAL OF 3D VIRTUAL ENVI- RONMENTS}

Abstract: 3D virtual environments are advanced technology systems, with some potentialities in the teaching and learning process.

In recent years, different institutions have promoted the acquisition of XXI century skills. Competences such as initiative, teamwork, creativity, flexibility or digital literacy.

Multi-user virtual environments, sometimes called virtual worlds or 3D simulators, are immersive, interactive, customizable, accessible and programmable systems. This kind of environments allow to design educational complex activities to develop these key competences. For this purpose it's necessary to set an appropriate teaching strategy to put this knowledge and skills into action, and design suitable mechanisms for registration and systematization.

This paper analyzes the potential of these environments and presents two experiences in 3D virtual environments: (1) to develop teamwork and self-management skills, and (2) to assess digital literacy in preservice teachers.

Key words: 3D Virtual Environments; Virtual Worlds; key competences; digital competence; team work; self-management.

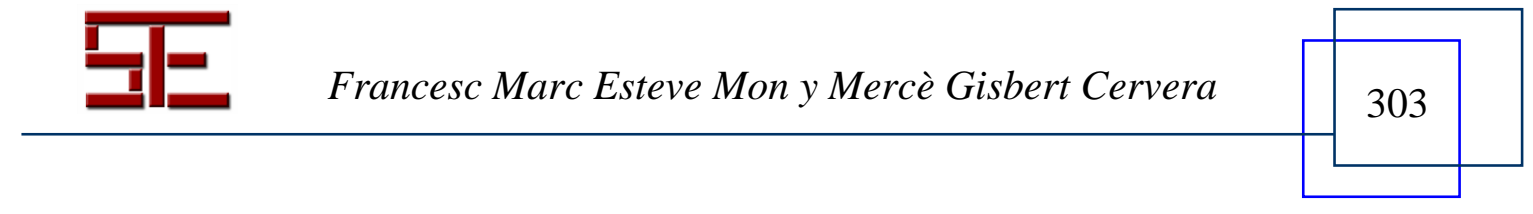




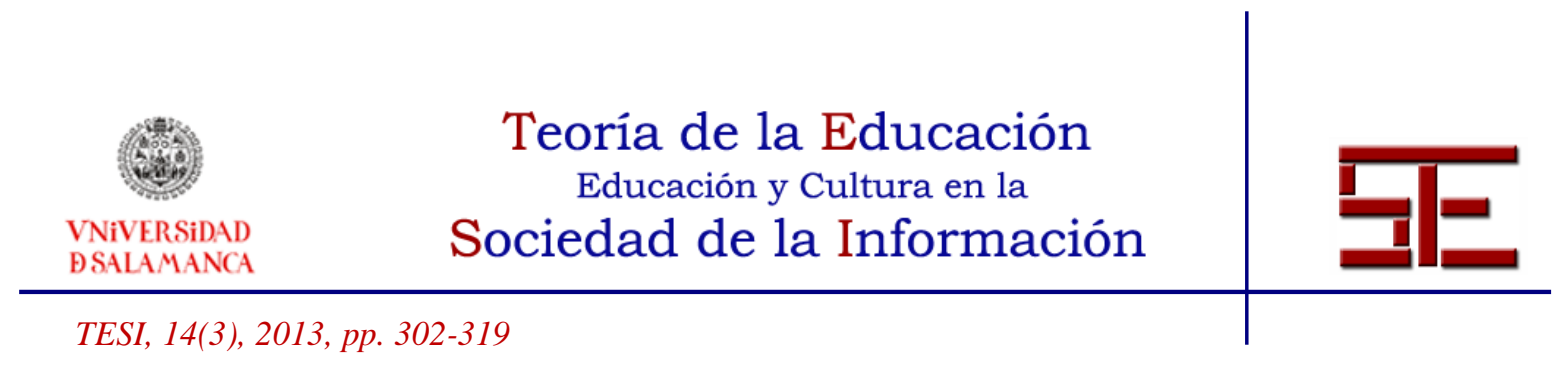

\section{EXPLORANDO EL POTENCIAL EDUCATIVO DE LOS ENTORNOS VIR- TUALES 3D}

Fecha de recepción: 31/10/2013; fecha de aceptación: 20/11/2013; fecha de publicación: 30/11/2013

Francesc Marc Esteve Mon

francescmarc.esteve@urv.cat

Universitat Rovira i Virgili,

Mercè Gisbert Cervera

merce.gisbert@urv.cat

Universitat Rovira i Virgili

\section{1.- INTRODUCCIÓN}

Las tecnologías de la información y la comunicación (TIC), que en estas últimas décadas se han incorporado en prácticamente todos los aspectos de nuestra vida, han propiciado no sólo una transformación de la economía global, sino un importante cambio en la forma de comunicarnos e interactuar con los demás, así como acceder a la información y al conocimiento, de manera permanente a lo largo de la vida (Castells, 1997; Fundación Telefónica, 2011).

Nuevos procesos, nuevos entornos y dispositivos, y nuevos perfiles de usuarios, que con la reciente expansión de la web 2.0, así como el impulso de la tecnología y los dispositivos móviles, sigue dando nuevos pasos, en todas las áreas de la sociedad y en todas las franjas de edad. También en el área educativa (Esteve, 2009).

A finales de 2012, la Comisión Europea presentó la comunicación titulada "Rethinking Education" en la que trata de recoger los principales retos y prioridades que deben abordar los estados en materia educativa. Tal y como afirma el documento (Comisión Europea, 2012), Europa sólo podrá reactivar el crecimiento apostando por la educación, y plantea, como uno de los principales retos, el fomento de las competencias transversales y básicas para el siglo XXI, destacando especialmente la iniciativa y el espíritu emprendedor, la competencia digital y los idiomas.

Como destaca la Comisión Europea (2007), el aprendizaje permanente se ha convertido en una necesidad para todos los ciudadanos. Resulta necesario la adquisición de una serie de conocimientos, capacidades y actitudes básicas para la realización personal, la inclusión social, la ciudadanía activa y la empleabilidad en este nuevo marco. En palabras de Ján Figel', responsable de educación de la Comisión Europea (2007, 1), "los sistemas de educación y formación deberían promover el desarrollo de dichas competencias entre todos los jóvenes".

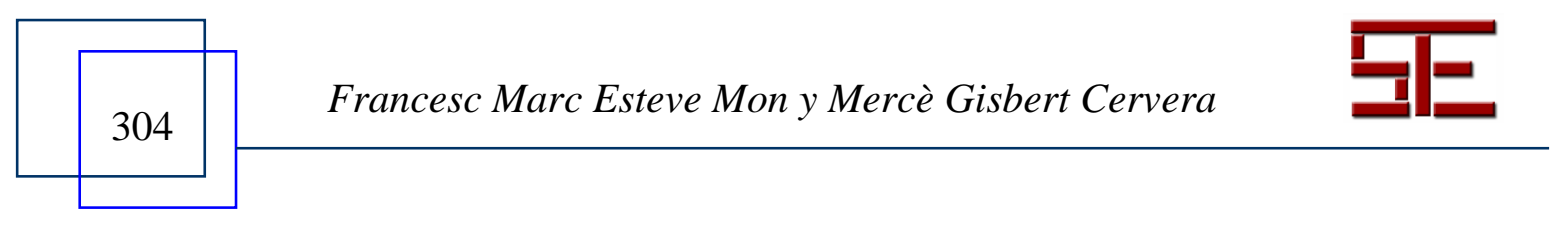




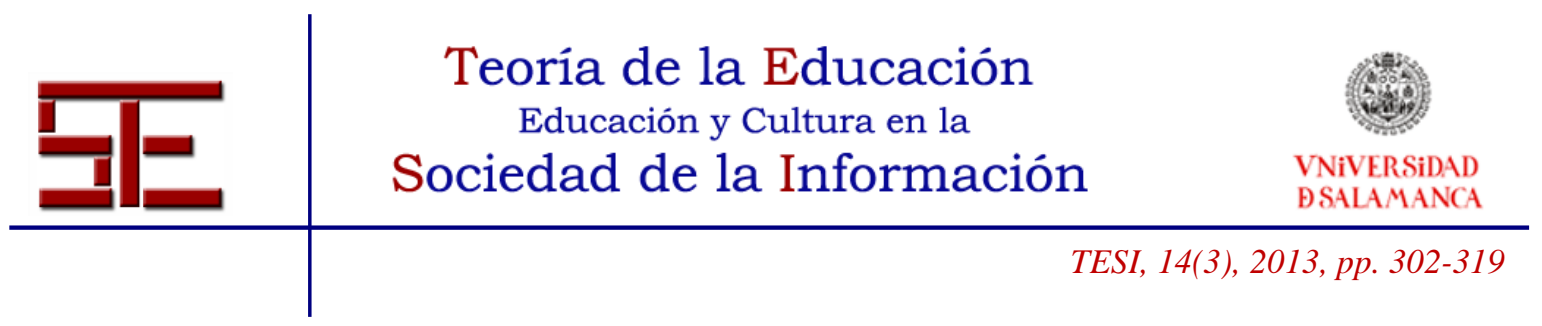

Nuevas competencias para el siglo XXI, en un escenario de aprendizaje permanente, flexible y abierto, que como ya estamos viendo en estos últimos años, está dando lugar a nuevas iniciativas como, por ejemplo, la creación de curso masivos en línea (MOOCs, según sus siglas en inglés), la redefinición los PLEs (entornos personales de aprendizaje) o la proliferación de múltiples tecnologías emergentes, como el mobile learning, la realidad aumentada, o los mundos virtuales 3D, que vislumbran nuevos ecosistemas tecnológicos de aprendizaje (Johnson, Adams y Cummins, 2012).

En estos últimos se centra el presente artículo, destacando el potencial educativo de estos entornos inmersivos y multiusuarios, que siguiendo el modelo OCDE (2005) para el desarrollo de las competencias clave, permite el aprendizaje autónomo y multidisciplinar, la colaboración con los demás, y la interacción en un entorno tecnológico avanzado.

\section{2.- NUEVOS ENTORNOS Y NUEVAS COMPETENCIAS PARA UN NUEVO PERFIL DEL ESTUDIANTE}

Resulta habitual, especialmente entre los más jóvenes, el uso diario de herramientas tales como los teléfonos móviles, Internet, los videojuegos, etc. Según la Fundación Telefónica (2009), casi el 90\% de los niños juega de forma habitual a videojuegos, o del mismo modo, el $80 \%$ de los adolescentes dispone de teléfono móviles, siendo ésta una de las tecnologías que más en auge ha estado desde finales de los 2.000 hasta la actualidad.

Algunos autores (Prensky, 2001; Tapscott, 1998; Oblinger y Oblinger 2005) hablan de que las características de los más jóvenes han cambiando radicalmente en los últimos años. Representan la primera generación que ha crecido rodeada de tecnologías, tales como Internet, los videojuegos o los teléfonos móviles, y por ende, poseen ciertas características y habilidades respecto a las TIC que los diferencian de las generaciones anteriores. Estos autores utilizan diferentes nombres o etiquetas para denominarlos: nativos digitales, generación Net, aprendices del nuevo milenio, generación Y, etc. Tapscott (1998 y 2009) utiliza el término Generación Net, para denominar a esa generación "bañada en bits", y los define especialmente por su curiosidad, independencia, apertura emocional e intelectual, libertad de expresión, grado de innovación, o por la necesidad de inmediatez, entre otras características.

Más allá de la denominación y la posición que tomemos ante este nuevo perfil de estudiantes, no podemos obviar las consecuencias de esta reciente revolución tecnológica. Como afirman Cabero y Llorente (2006), en este nuevo entramado, se hace necesario que los sujetos estén capacitados para movilizar y utilizar las nuevas herramientas de comunicación que tienen a su disposición en la sociedad del conocimiento. Y por ende, la sociedad, y en especial las instituciones educativas han de ser conscientes de esta

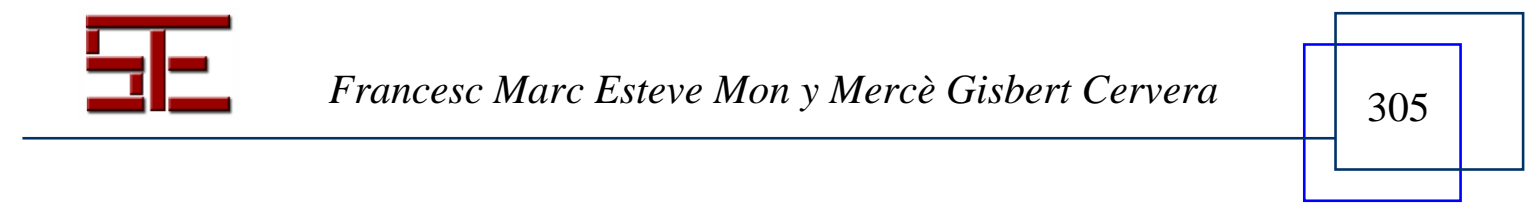




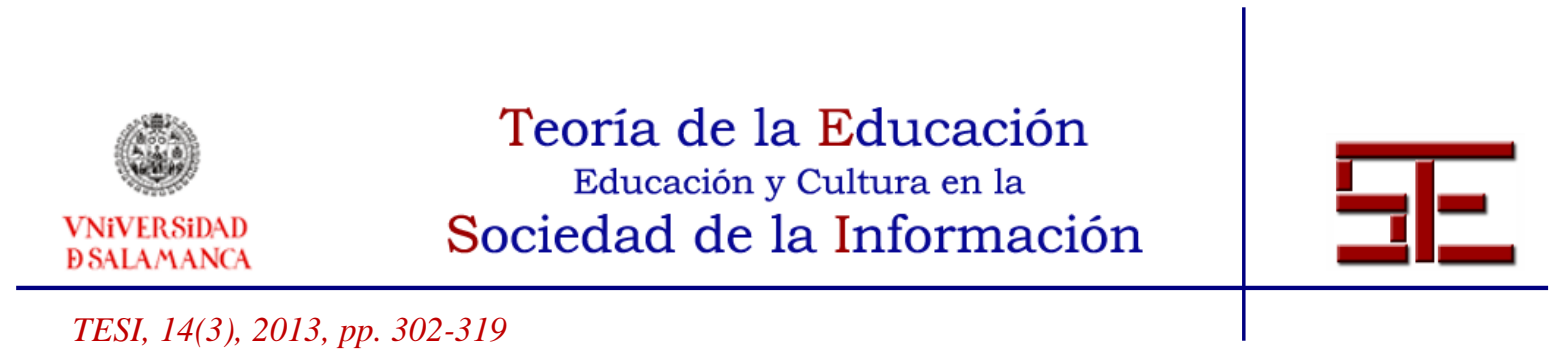

serie de cambios globales y replantearse cuáles son los aspectos clave para la educación en este nuevo contexto.

Desde hace unos años, numerosas instituciones públicas y privadas han impulsado una revisión y reorganización de las prioridades educativas para el siglo XXI con motivo, según éstas, de la necesaria adaptación a los nuevos retos que nos plantea hoy en día la sociedad. Como posteriormente analizaremos con más detalle, este nuevo discurso expone la necesaria adquisición de una serie de competencias clave para hacer frente a los nuevos tiempos, y enfatiza la importancia del aprendizaje a lo largo de la vida.

En 1999, la OCDE se planteó frente a esta situación, la necesidad de analizar los niveles de destrezas de la población y los efectos de la educación, desarrollando un marco común para la identificación y definición de las competencias para el siglo XXI (Salganik et al., 1999). En 2006, la Unión Europea anima a desarrollar la oferta de Competencias clave para el aprendizaje permanente (Unión Europea, 2006). Según define el propio documento (p.13), "las competencias clave son aquéllas que todas las personas precisan para su realización y desarrollo personales, así como para la ciudadanía activa, la inclusión social y el empleo", sin embargo, competencias clave con un enfoque muy clásico y de tipo disciplinar, en contraposición con otros enfoques (Pérez Gómez, 2007).

El modelo norteamericano Framework for 21st Century Learning desarrollado por el Partnership for 21st Century Skills (2009), también conocido como P21, ofrece una visión holística y sistémica de la educación para el siglo XXI, definiendo una serie de habilidades como la creatividad, el pensamiento crítico, la flexibilidad, la iniciativa, la productividad o las habilidades tecnológicas, informacionales y multimedia, esenciales para este nuevo siglo.

Esta última competencia, la competencia digital, entendida como el conjunto de habilidades y conocimientos tecnológicos, informacionales, comunicativos y multimedia que cualquier estudiante debe desarrollar y utilizar, de manera creativa, crítica, reflexiva, ética, y efectiva (Livingstone, 2004; Hobbs, 2010), es una de las competencias más reiteradas en la mayoría de los marcos de habilidades clave para el siglo XXI (Esteve, Adell y Gisbert, 2013).

Para ello, es necesario, como plantea Erstad (2010), avanzar hacia (1) una nueva cultura de la participación, con nuevas formas y vías de interacción y colaboración, (2) nuevos sistemas de acceso a la información, (3) nuevas posibilidades de comunicación, explorando nuevos dispositivos, entornos y canales, y (4) nuevas formas de producción de contenido, haciendo uso del potencial de la red, y de las múltiples herramientas digitales.

Siguiendo el estudio impulsado por el Instituto de Prospectiva Tecnológica de la Unión Europea (Redecker, 2013), se abre un interesante horizonte para el desarrollo y la eva-

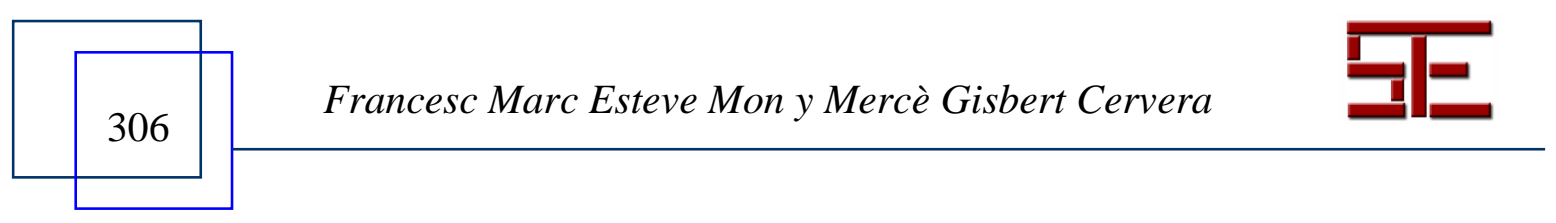




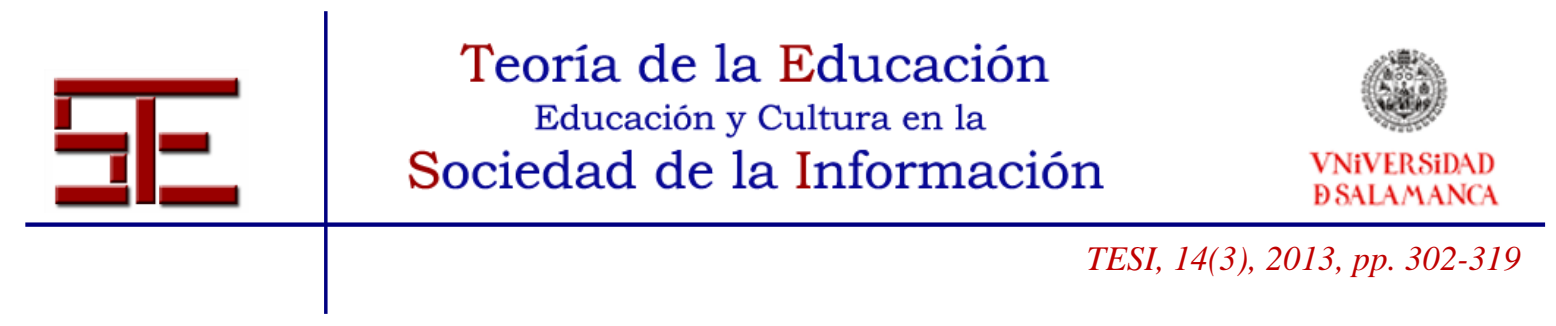

luación de las competencias clave del siglo XXI a través de diferentes entornos tecnológicos (figura 1).

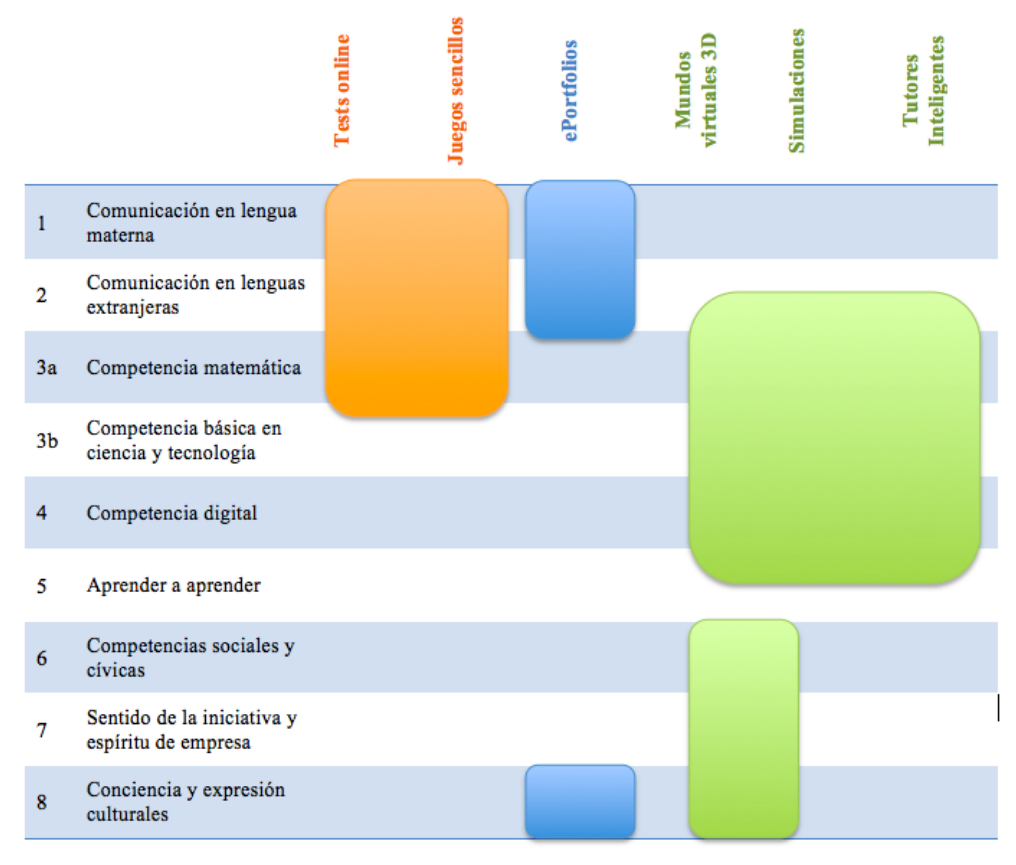

Figura 1: Potencial educativo de los entornos tecnológicos para la evaluación de competencias clave del siglo XXI (Fuente: Adaptación de Redecker, 2013)

Como vemos en la figura 1, resulta especialmente idóneo el desarrollo de estas competencias a través de sistemas tecnológicos como los portfolios digitales, los mundos virtuales, los juegos digitales, o los simuladores 3D.

\section{3.- LOS ENTORNOS VIRTUALES 3D EN EDUCACIÓN}

Los entornos virtuales 3D, como por ejemplo Second Life u OpenSimulator, son comunidades online que simulan espacios físicos en tres dimensiones, reales o no, y que permiten a los usuarios, a través de sus avatares, interactuar entre sí, y utilizar, crear e intercambiar objetos. Atkins (2009) apunta las siguientes características de estos entornos:

-De inmersión. Más allá de las sencillas simulaciones, de aplicaciones o experiencias concretas, estos escenarios permiten la inmersión espacial de los usuarios, dando la sensación de estar presente en un entorno simulado, que ellos sienten como real.

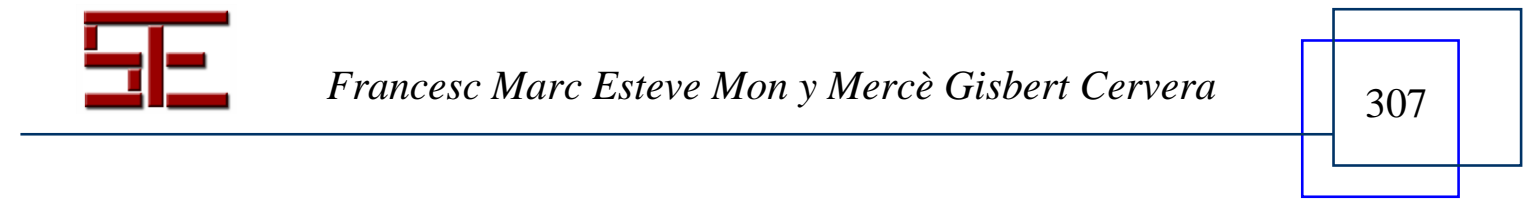




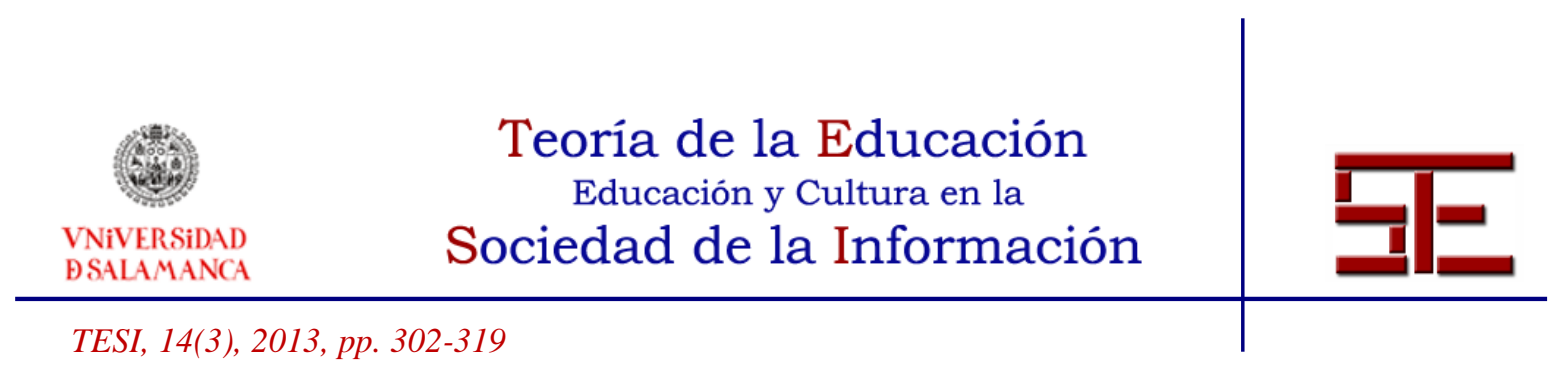

-Interactivos. Estos entornos ofrecen un espacio interactivo, en tiempo real, que incluye la comunicación por voz y texto, así como la facilidad de visualizar y escuchar elementos multimedia por streaming. Esta capacidad potencia considerablemente la sensación de inmersión, y las posibilidades de estas experiencias.

-Personalizables. Otra de las características de estas herramientas es la posibilidad de personalización que ofrece, permitiendo construir y diseñar, de forma individual o colectiva, los escenarios y los objetos de estos mundos virtuales.

-Accesibles. Otra característica importante, tanto de SecondLife como sobre todo en OpenSimulator, es su disponibilidad y acceso de manera gratuita. Otros entornos requieren de una suscripción de pago, mientras que éstos pueden usarse de manera gratuita, abierta y accesible.

-Programables. Como menciona Atkins (2009), para algunas personas del mundo de los videojuegos éstos entornos carecen de interés a simple vista, ya que cuando uno accede no tiene unas instrucciones concretas qué hacer en ese entorno, como pasa en los juegos. SecondLife u OpenSimulator no son juegos, sino que son motores de juegos. No tienen reglas predefinidas, ni misiones, ni sistemas por puntos que ir cumpliendo, sino que permite a los usuarios crear sus objetos y escenarios, y programar en ellos sus scripts y sus propias normas y objetivos.

Todo ello favorece la creación de entornos con reglas físicas alternativas, el intercambio síncrono y asíncrono, la alteración de la compresión del tiempo (Allen y Demchack, 2011), y numerosas potencialidades que pueden resultar muy interesantes para prácticas e investigaciones educativas (Gisbert et al., 2011). Frente a ello, también se han detectado algunos inconvenientes que pueden dificultar su extensión, como la poca familiariedad con el entorno y la elevada curva de aprendizaje, el elevado nivel de requisitos técnicos para su funcionamiento o el grado de fiabilidad, confianza y seguridad del sistema (Atkins, 2009). Aspectos, todo ellos, que se deberá tener en cuenta a la hora de diseñar actividades de carácter educativo.

En estos años, diferentes universidades han empezado a utilizar estas herramientas a nivel educativo. Como apuntan diferentes autores, es necesario explorar su uso más allá de fines publicitarios, clases magistrales y conferencias, potenciando actividades de aprendizaje más centradas en el estudiante (Alrayes y Sutcliffe, 2011). Allen y Demchak (2011) exponen que estos entornos virtuales 3D pueden utilizarse para las siguientes finalidades: presencia virtual de instituciones, procesos educativos, entrenamiento, planificación de actividades, ensayos, análisis de experiencias, experimentación, pruebas y evaluaciones, y actividades de concienciación.

Como vemos en las investigaciones de Clarke y Dede (2007 y 2009), y más recientemente Code, Clarke, Zap y Dede (2011), Alice in Wonderland, River City o VPA han sido experiencias muy interesantes en el uso de estos entornos virtuales multiusuario

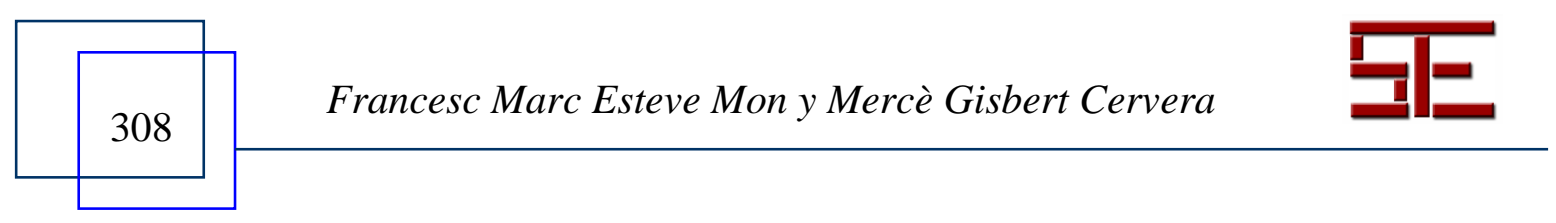




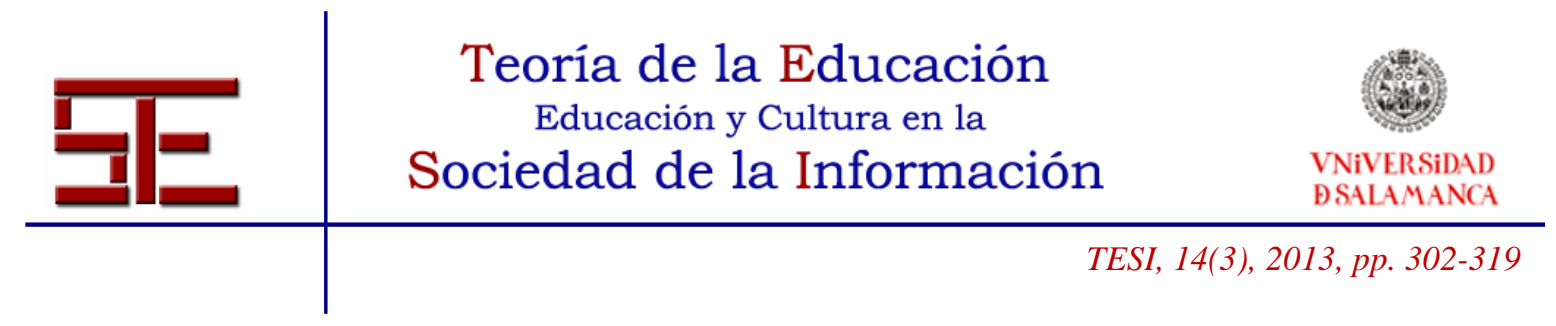

(MUVEs), realizados mayormente en Estados Unidos y en los que han participado más de 15.000 estudiantes de distintos niveles educativos, y más 250 profesores, colaborando, comunicándose e interactuando en entornos inmersivos, a través de esta tecnología avanzada.

\section{4.- HACIA UNA NUEVA ESTRATEGIA DIDÁCTICA EN ENTORNOS VIR- TUALES 3D}

El desarrollo y la evaluación de competencias, como parte intrínseca del proceso de enseñanza-aprendizaje, requiere de situaciones y estrategias formativas adecuadas, no sólo centradas en la adquisición y memorización de conceptos y contenidos sino en la puesta en acción de dichos conocimientos, junto con habilidades, destrezas y actitudes, de manera efectiva y eficiente, ante una determinada situación (De la Orden, 2011).

Prades (2004), siguiendo los trabajos de Miller (1990), afirma que los criterios más relevantes para seleccionar la estrategia de aprendizaje y el instrumento de evaluación más adecuado son la coherencia con los objetivos de aprendizaje, la validez y la fiabilidad, y otras cuestiones relativas al impacto en el aprendizaje y el coste en términos de tiempo y recursos. La pirámide de Miller (1990), según Prades (2004), puede ser una manera útil para elegir estrategias didácticas y evaluativas, de manera coherente con los objetivos de aprendizaje determinados (figura 2).

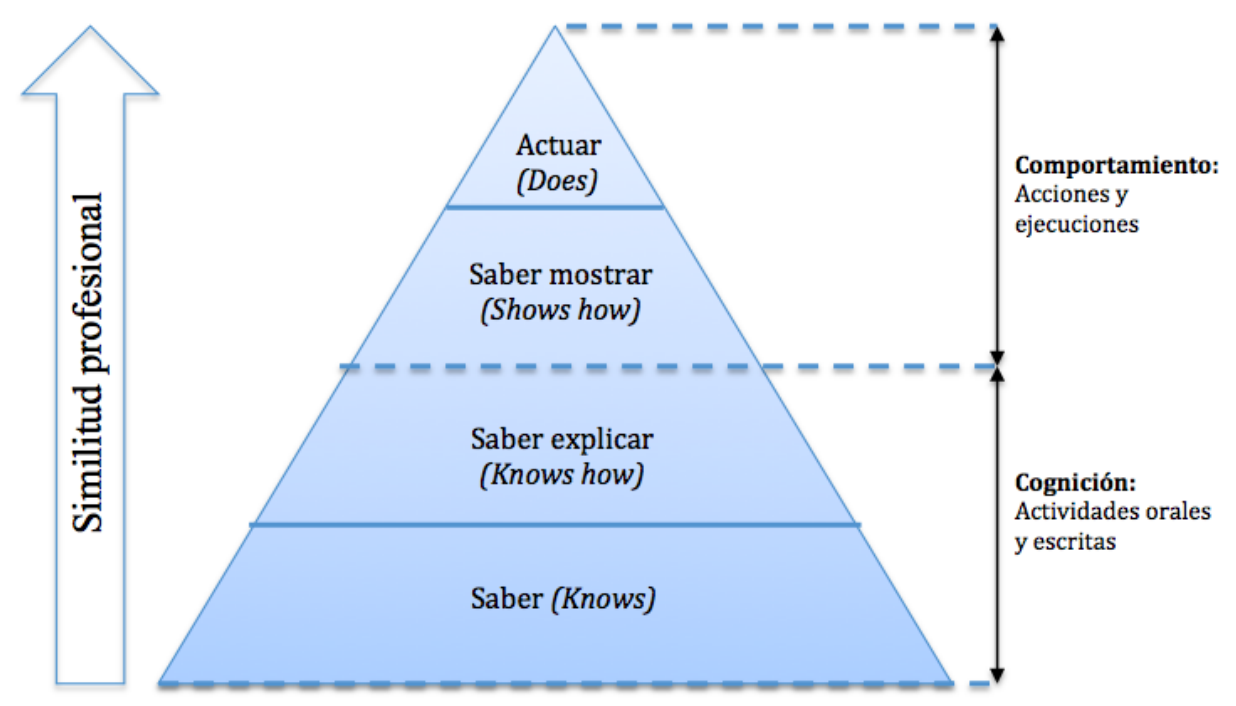

Figura 2: Estrategias didácticas según la Pirámide de Miller (Fuente: Adaptación de Prades, 2004)

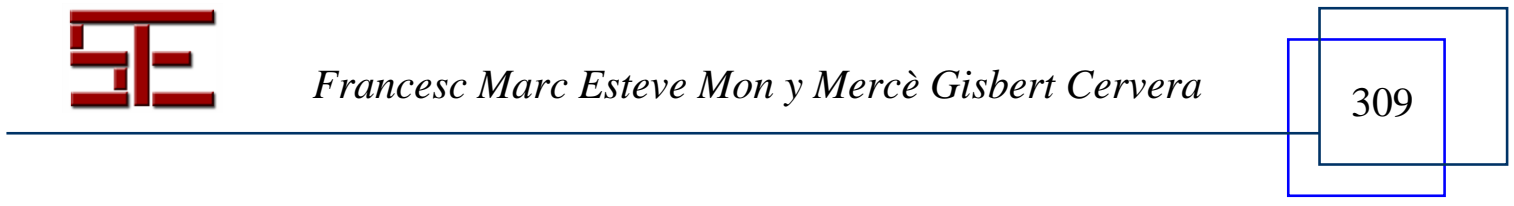




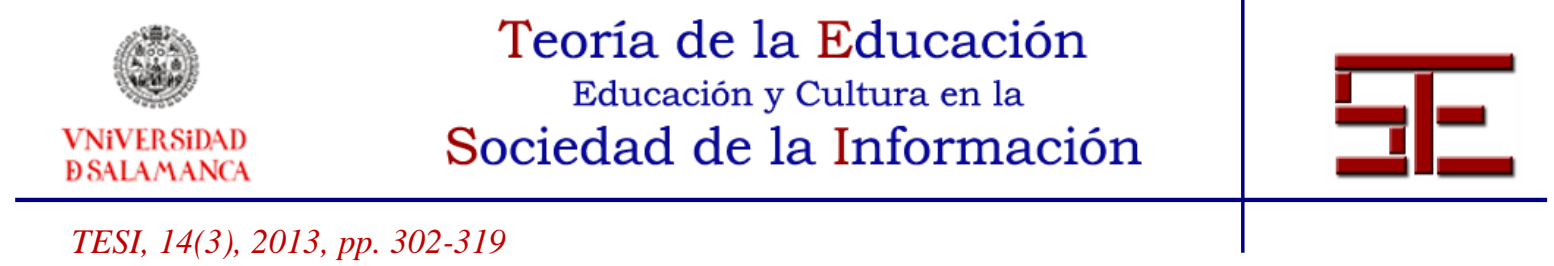

Estrategias como el Aprendizaje Basado en Problemas, permiten poner en acción, tales competencias (Vosinakis et al., 2011). Asimismo, como destaca Prades (2004), los cuestionarios, los tests o las pruebas de respuesta corta son instrumentos adecuados para la evaluación de actividades que requieran la comprensión, la memorización o aplicación de conceptos, al implicar habilidades cognitivas, mientras que la elaboración de productos, los portafolios, los dossieres de aprendizaje o entornos más complejas que permitan la acción, pueden ser muy adecuadas para la evaluación de ejecuciones y comportamientos.

Según De la Orden $(2011,12)$, "el dominio de una competencia, pues, únicamente se manifiesta en el desempeño de la función o rol correspondiente y, en consecuencia, sólo puede ser constatado y confirmado contrastando el éxito de dicho desempeño con un criterio previamente establecido".

Para tener constancia de la evolución del proceso de aprendizaje del estudiante es necesario hacer uso de una serie de técnicas de evaluación que, a través del uso de determinados instrumentos, permitan recoger información y evidencias. La utilidad de un instrumento en el ámbito de la evaluación radica en su potencialidad para poner de manifiesto aquello que pretende evaluar y su potencialidad real de ser bien utilizado (Salinas, 2002). Para ello, será necesario operativizar la definición de estas competencias, definiendo sus objetivos de aprendizaje (Prades, 2005), y teniendo en cuenta el saber (conocimientos), el saber hacer (habilidades y destrezas), y el saber ser y estar (actitudes), que ya mencionaba Delors (1996). Uno de los instrumentos que pueden ser útiles para este tipo de evaluación son las rúbricas, instrumentos en los que se definen algunos criterios de valoración y desempeño de un producto, un proyecto, o una tarea en varios niveles progresivos de rendimiento o ejecución (Blanco, 2008; Gil, 2007), y que pueden utilizarse para valorar, tutorizar y evaluar distintos niveles de desempeño y dominio de competencias (Raposo y Martínez, 2011; Bilbatua y Egizabal, 2010).

Los entornos virtuales 3D, que hemos expuesto en el punto anterior, son espacios de tecnología avanzada que permiten el desarrollo de estrategias formativas, tales como las actividades basadas en problemas, que permiten poner en acción aquellas competencias que se pretenden desarrollar (Beaumont et al., 2012; Rice, 2009; Vosinakis et al., 2011). Asimismo, permite una evaluación efectiva del desempeño de tareas complejas, a través del establecimiento de rúbricas y la sistematización de los procesos de registro y codificación de las actividades (Code, Clarke, Zap y Dede, 2011).

\section{5.- ALGUNAS EXPERIENCIAS EDUCATIVAS EN ENTORNOS VIRTUALES 3D}

A continuación describimos dos experiencias diferentes que hemos realizado en los últimos años en nuestra universidad para el desarrollo y la evaluación de diferentes com-

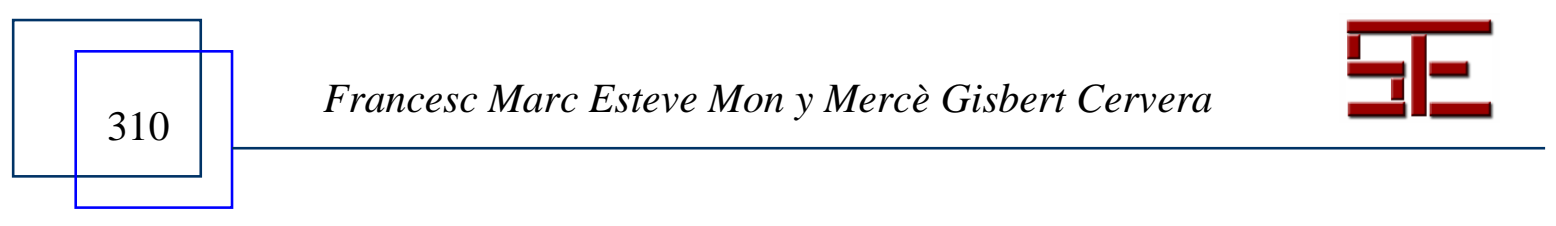




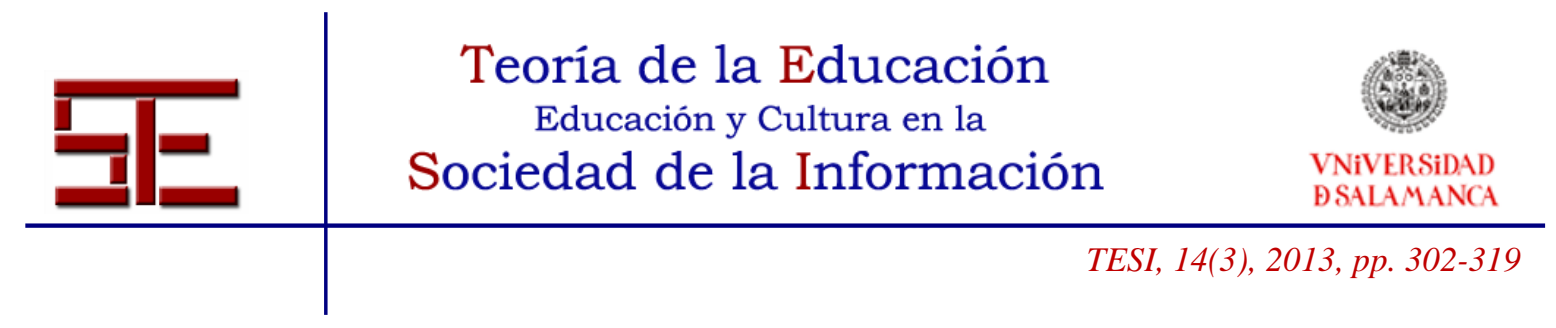

petencias clave para el siglo XXI, utilizando entornos virtuales 3D. Esta es una universidad española de tamaño medio (12,000 estudiantes de grado, 900 de master y alrededor de 900 profesores); con un campus distribuido en diferentes sedes y con un amplio abanico de titulaciones distintas.

En estos estudios se utilizó la herramienta OpenSimulator, un servidor de plataformas 3D, de código abierto, que puede utilizarse para la creación de entornos o mundos virtuales, accesibles a través de una variedad de clientes y en múltiples protocolos. Para ello, y como veremos de forma más detallada en los siguientes apartados, se utiliza un módulo, llamado Sloodle, que permite integrar OpenSimulator con el sistema de gestión del aprendizaje o LMS, Moodle. Este módulo incluye diferentes componentes para el diseño de actividades, tales como preguntas de elección dicotómica, canales de chat para interactuar entre los diferentes avatares, posibilidad de visualizar información multimedia, etc., interactuando con su personaje, con sus compañeros o con los objetos de su entorno, y quedando todo ello registrado en su actividad en Moodle.

\subsection{Experiencia 1: SIMUL@, un mundo virtual para el desarrollo de la competencia de trabajo en equipo y autogestión}

En esta primera experiencia participaron 72 estudiantes $(72,2 \%$ mujeres y $27,8 \%$ hombres) de una edad media de 24,13 (sd=6,23) correspondientes a titulaciones de Grado de Educación y Magisterio (Educación Infantil: 65,2\%; Educación Primaria: 2,8\%; Educación Física: 15,3; Pedagogía y Educación Social: 4,2\%) y Máster de Dirección de Marketing $(12,5 \%)$. Los estudiantes participaron de manera voluntaria y la experiencia tuvo lugar en el contexto de una asignatura correspondiente al currículum ordinario de las titulaciones, durante el curso 2010-2011, y en el marco del proyecto Simul@ (Ref.: EDU2008-01479), del Plan Nacional de I+D+I 2008-2011 del Ministerio de Ciencia e Innovación.

Se diseñó una propuesta didáctica centrada en el desarrollo y la evaluación de las competencias transversales de autogestión y trabajo en equipo, todo ello desarrollado dentro de un mundo virtual 3D. Para ello, se categorizaron las actividades a través de rúbricas, adaptándolas al contex to de la asignatura.

En dicho escenario se representó una serie de casos, reales, que los estudiantes debían realizar construyendo y defendiendo un producto determinado: (1) la organización de unas jornadas para un encuentro de escuelas rurales, (2) la organización de unas olimpiadas escolares, y (3) la participación en una feria profesional (figura 3).

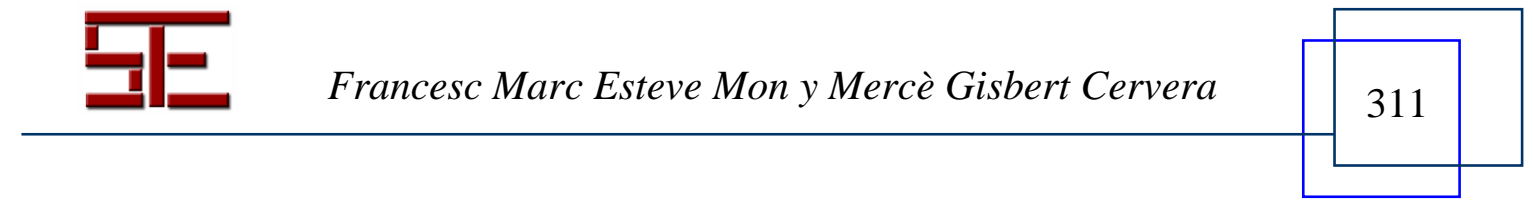



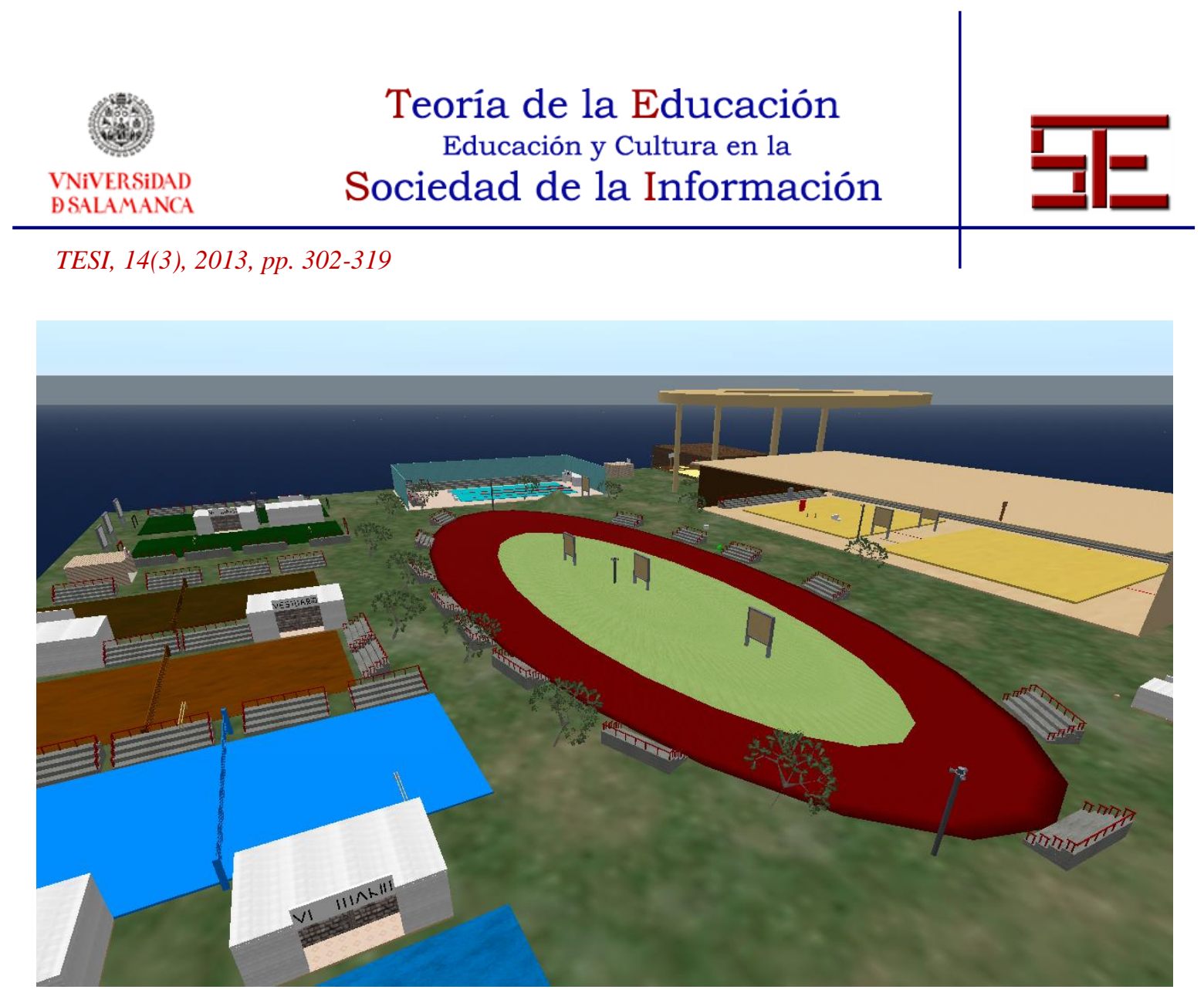

Figura 3: Imagen de las instalaciones para las olimpiadas escolares

Cada escenario contó con un espacio común, llamada isla general, donde realizar pruebas y adquirir recursos, y una isla vacía para cada grupo, donde construían sus proyectos. El entorno desarrollado registraba el chat entre los usuarios, los objetos utilizados, y el producto desarrollado por los estudiantes.

En la primera fase, la fase de desarrollo, los estudiantes debían realizar diferentes tareas para la obtención de puntos que podían canjear por objetos extra para la construcción de su proyecto. Todas estas actividades estaban relacionadas con las rúbricas de cada una de las asignaturas. La tipología de las actividades según las rúbricas es: (1) Organización personal, (2) Organización espacial, (3) Organización documental, (4) Elaboración de listados, (5) Elaboración de presupuestos, (6) Elaboración de calendarios, (7) Programación y anticipación, y (8) Explicación y argumentación de ideas.

En la segunda fase, la construcción, los estudiantes debían organizar su espacio y construir su proyecto en su isla. Para ello podían crear objetos, utilizar y modificar los existentes en los distintos dispensarios y gestionar los puntos obtenidos en la fase de desarrollo. Como resultado de esta fase, cada grupo de estudiantes debía presentar su isla como un producto para ser evaluado delante de un comité.

En la tercera fase, la evaluación, los estudiantes presentaban su isla delante del comité evaluador y el resto de equipos, justificando las características y fortalezas de su proyecto respecto al contexto de la asignaturas, y exponían las actividades realizadas en la fase

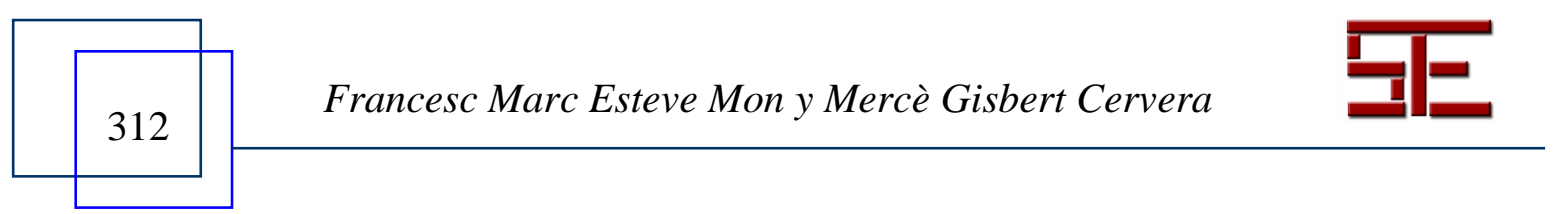




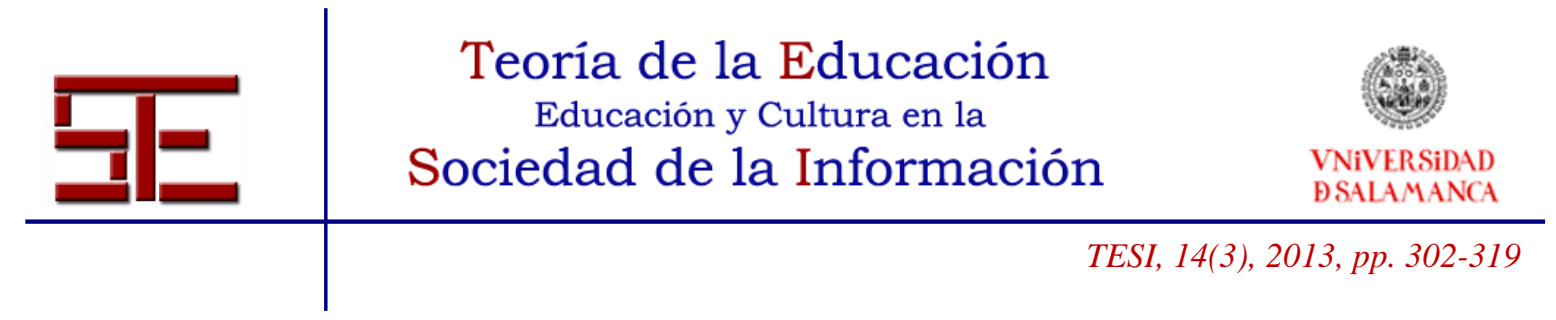

de desarrollo. A continuación, se realizó una votación donde el resto de equipos valoró su trabajo, así como se administraron una serie de cuestionarios de autopercepción acerca de su autogestión, de su trabajo en equipo y del uso del entorno virtual 3D.

Experiencia 2: Evaluación de la competencia digital de los futuros maestros en entornos $3 D$

Esta segunda experiencia se empezó a desarrollar en el curso 2012-2013, encontrándose actualmente en fase de aplicación y validación. El objetivo principal es diseñar y crear un entorno virtual 3D para el desarrollo y la evaluación de la competencia digital en estudiantes del grado en educación infantil y primaria.

Para ello, en una primera fase se profundizó en el concepto de competencia digital docente, y se operativizó a través de los indicadores y estándares de la International Society for Technology in Education (ISTE, 2008). Tales indicadores se distribuyeron de manera conceptual en tres posibles escenarios: (1) el aula, espacios y recursos (2) diseño de actividades didácticas, y (3) recursos para el aprendizaje permanente. Tras ello, se diseñaron y desarrollaron a nivel tecnológico los distintos escenarios (figura 4).
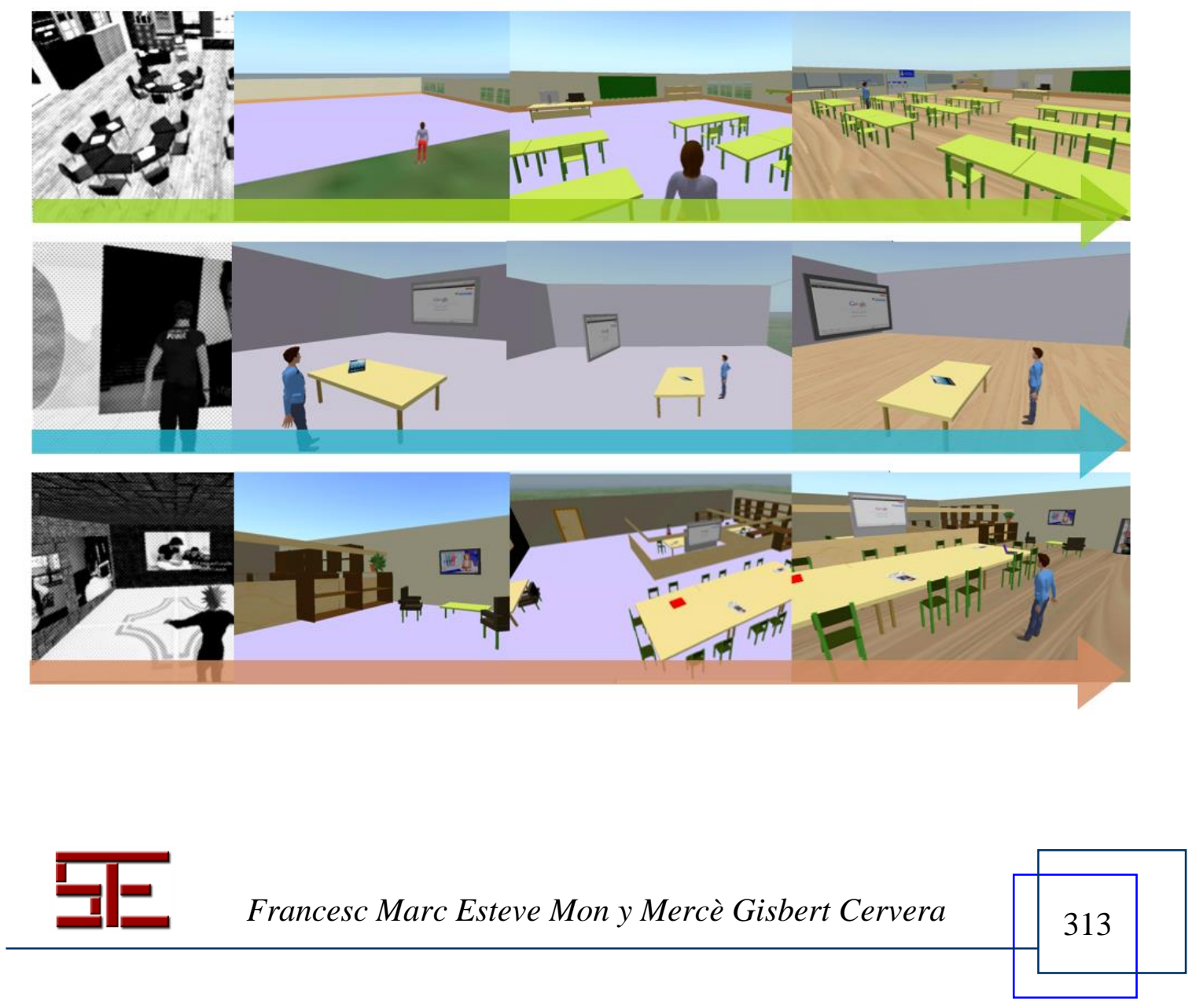


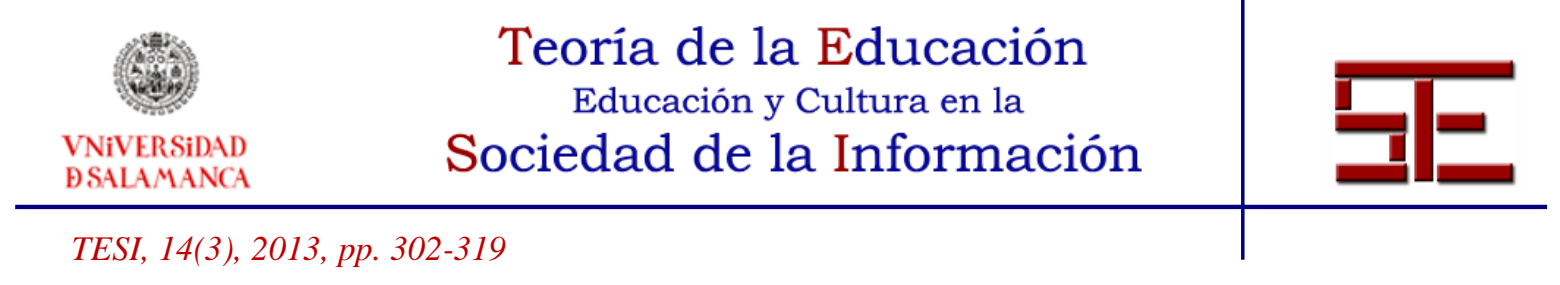

Figura 4: Proceso de diseño y desarrollo de los tres escenarios de evaluación de la competencia digital

Cada escenario cuenta a su vez con una serie de instrucciones precisas que indican al estudiante qué debe realizar en cada uno de ellos, así como información adicional en los diferentes objetos con los que interactúa. A continuación podemos ver una muestra del texto inicial del primer escenario:

Bienvenidos/as a la escuela "La Muntanya". Nos encontramos en un aula de primaria, de un pequeño colegio rural. Ahora, los/as tutores/as sois vosotros/as. Explorad la clase, analizad sus características, buscad y revisad los documentos del centro y de la clase. Deberéis repensar, de forma consensuada, el espacio formativo del aula, su organización, y los recursos que vais a utilizar en función de las características del centro, del aula, de vuestros/as alumnos/as, y de vuestras intenciones pedagógicas.

Tendréis que tratar de utilizar los pocos recursos que ya hay en el aula. Y, además, podréis utilizar hasta un máximo de 2 recursos complementarios que encontraréis en el almacén de recursos.

Así que, explora el espacio y piensa las posibilidades Discute, a través del chat, la propuesta con los compañeros/as que encontrarás en el aula. Montad vuestra propuesta en el aula: reoganizad los espacios, moved los objetos, haced evidente que da respuesta a las necesidades expuestas anteriormente. Y para acabar, ve al buzón de entrega y explica, de manera individual y con todo tipo de detalles, la propuesta que habéis elaborado. Tenéis, 25 minutos!

Este instrumento de evaluación, desarrollado en un entorno 3D, se completa en un máximo de 90 minutos en un aula de clase con ordenadores (figura 5). Los estudiantes realizan una serie de actividades individuales, y otras grupales, llevándose a cabo una evaluación formativa acerca de su competencia digital, siguiendo los indicadores de ISTE (2008).

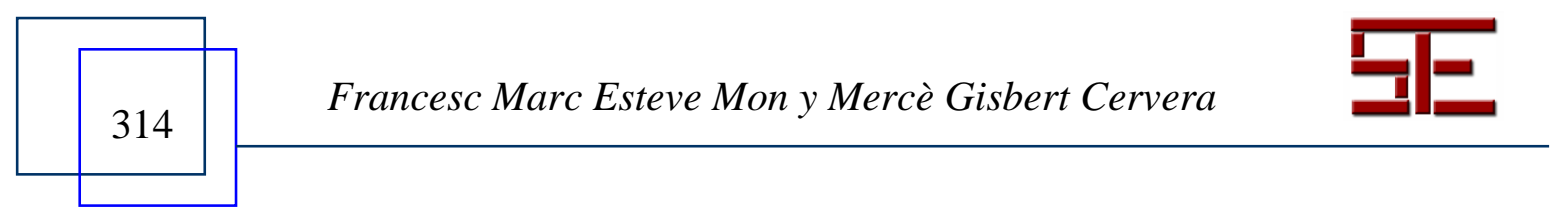



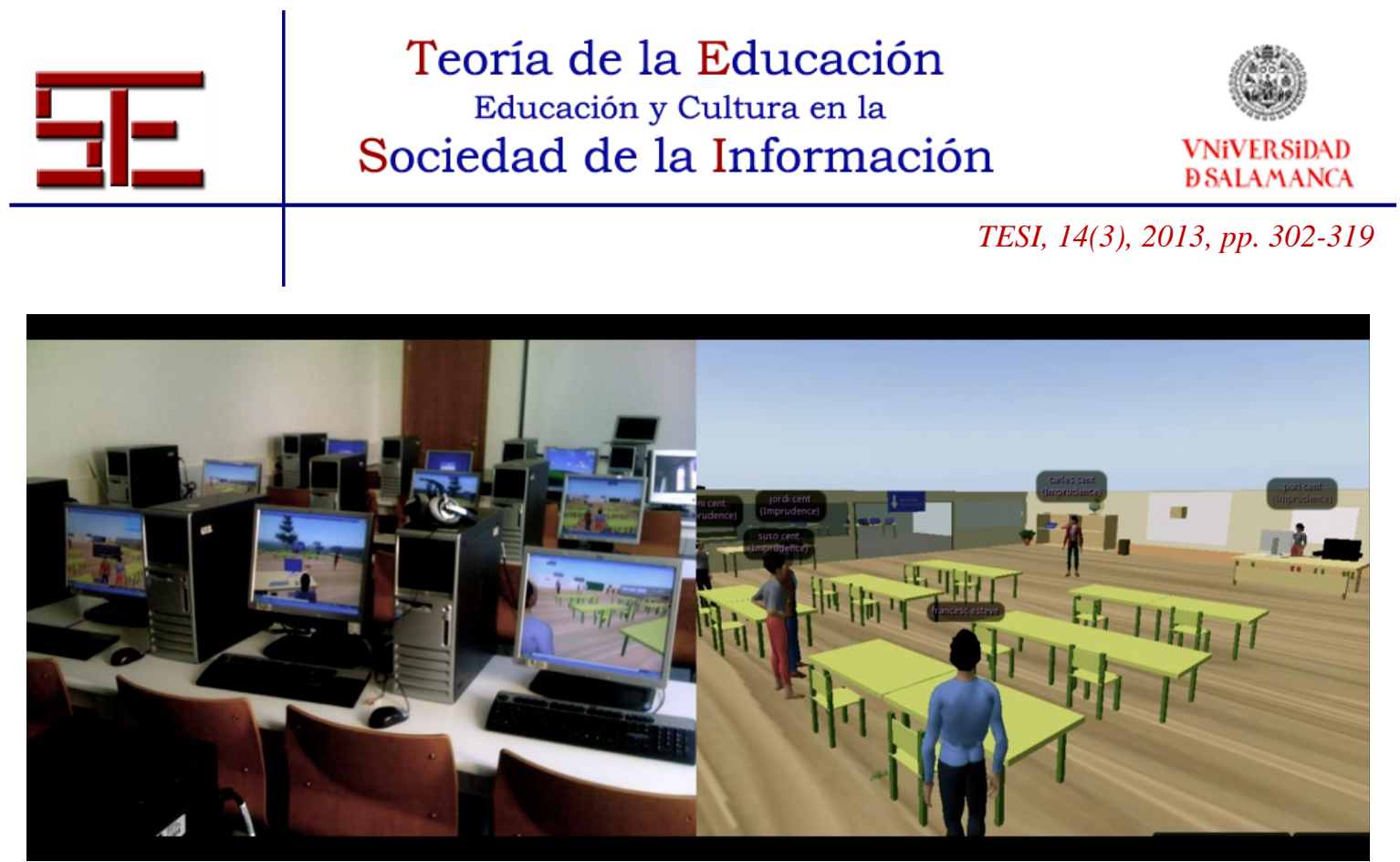

Figura 5: Aula presencial y aula virtual 3D para la evaluación de la competencia digital docente

\section{6.- A MODO DE CONCLUSIÓN}

Como hemos visto a lo largo del presente artículo, los entornos virtuales 3D, como Second Life u OpenSimulator, son espacios que permiten realizar una inmersión en un entorno simulado, interactuar con los otros usuarios, utilizar, crear e intercambiar objetos, y son personalizables y programables, según la finalidad deseada. Como platean diversos autores (Gisbert et al., 2011; Alrayes y Sutcliffe, 2011; Allen y Demchak, 2011), estos entornos tienen muchas potencialidades en el campo educativo, tanto para la realización de experiencias y prácticas formativas, como para actividades de experimentación, pruebas, simulaciones o actividades de trabajo en grupo, quedando registrada toda la actividad realizada en ellas, de manera rigurosa y sistemática (Clarke y Dede, 2007).

Estos ecosistemas tecnológicos avanzados pueden representar una interesante vía para el desarrollo y la evaluación de las competencias clave para el siglo XXI (Redecker, 2013). En las experiencias que nosotros hemos llevado a cabo, y que hemos expuesto anteriormente, los resultados han sido muy positivos, tanto en el rendimiento académico de las distintas materias y competencias, como especialmente en la motivación de los y las estudiantes. Cabe recordar que los estudiantes actuales, como veíamos en los primeros puntos, representan una generación que convive a diario con la tecnología y que la prefiere para sus procesos formativos (Prensky, 2001; Tapscott, 1999; Oblinger y Oblinger 2005). Por ende, estos entornos suscitan líneas de trabajo muy interesantes en estos campos.

En cuanto a la tecnología, los entornos 3D requieren de unas elevadas exigencias técnicas y gráficas, en constante renovación. Sin embargo, iniciativas de código abierto, co-

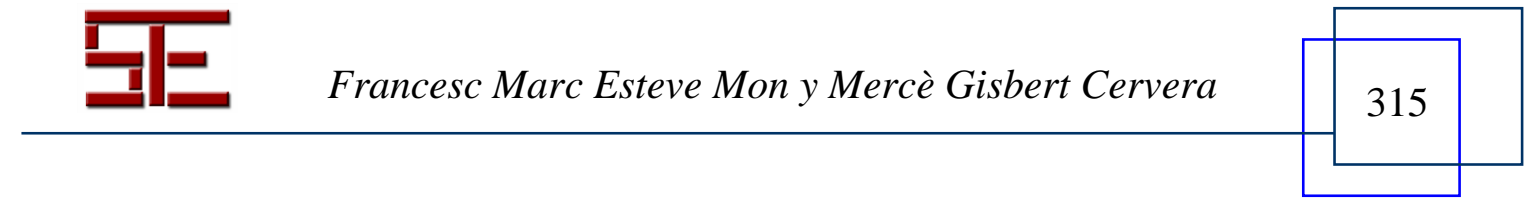




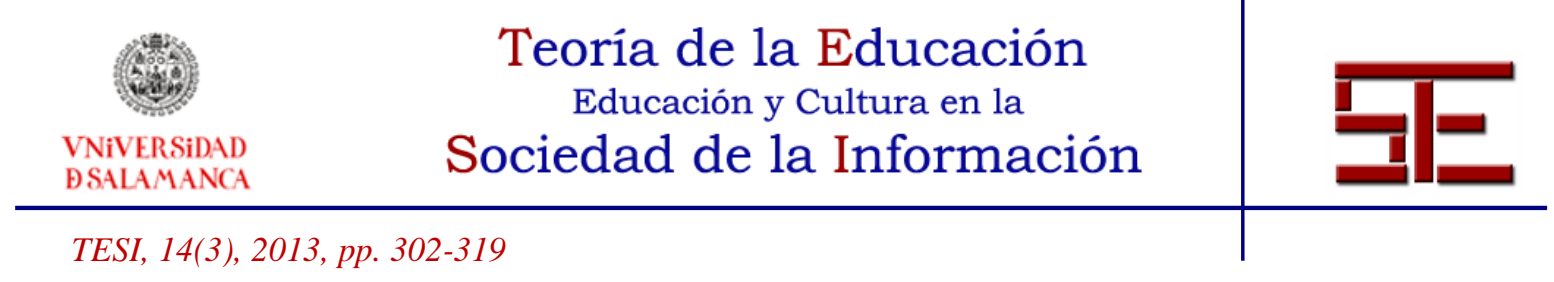

mo OpenSimulator, a pesar de sus potencialidades en algunos aspectos puede resultar obsoleto. Herramientas como por ejemplo Unity 3D, OpenWonderland y otros sistemas similares, ofrecen una buena oportunidad para el desarrollo de simuladores 3D y videojuegos de alta calidad tecnológica y gráfica, disponibles también en dispositivos móviles y tabletas, algo que sin duda vislumbra también nuevos horizontes de investigación.

\section{7.- BIBLIOGRAFÍA.}

Allen, P. D. y Demchak, C. C. (2011). Applied virtual environments: Applications of virtual environments to government, military and business organizations. Journal of Virtual Worlds Research, 4(1).

Alrayes, A. y Sutcliffe, A. (2011). Students' attitudes in a virtual environment (secondlife). Journal of Virtual Worlds Research, 4(1).

Atkins, C. (2009). Virtual experience: Observations on second life. In M. Purvis \& B. Savarimuthu (Eds.), Lecture Notes in Computer Science: Computer-Mediated social networking, pp. 7-17. Berlin: Springer.

Beaumont, C., Savin-Baden, M., Conradi, E., \& Poulton, T. (2012). Evaluating a second life problem-based learning (PBL) demonstrator project: What can we learn? Interactive Learning Environments, 1-1.

Bilbatua, M. y Egizabal, D. (2010). Las rúbricas en la reflexión sobre el trabajo en equipo., en Bujan, K. (Eds.). Seminario internacional Las rúbricas de evaluación en el desempeño de competencias: ámbitos de investigación y docencia, Universidad del País Vasco, San Sebastián (España).

Blanco, A. (2008). Las rúbricas: un instrumento útil para la evaluación de competencias, en La enseñanza universitaria centrada en el aprendizaje: estrategias útiles para el profesorado. Barcelona: Octaedro-ICE de la Universidad de Barcelona.

Cabero, J. y Llorente, M. C. (2006). La rosa de los vientos. Dominios tecnológicos de las TICs por los estudiantes. Sevilla, GID.

Castells. (1997). La era de la información: Economía, sociedad y cultura. La sociedad en red. Madrid: Alianza Editorial.

Clarke, J. y Dede, C. (2007). MUVEs as a powerful means to study situated learning. En Proceedings of the 8th iternational conference on computer supported collaborative learning (pp. 144-147).

Clarke, J. y Dede, C. (2009). Design for scalability: A case study of the river city curriculum. Journal of Science Education and Technology, 18(4), 353-365. 


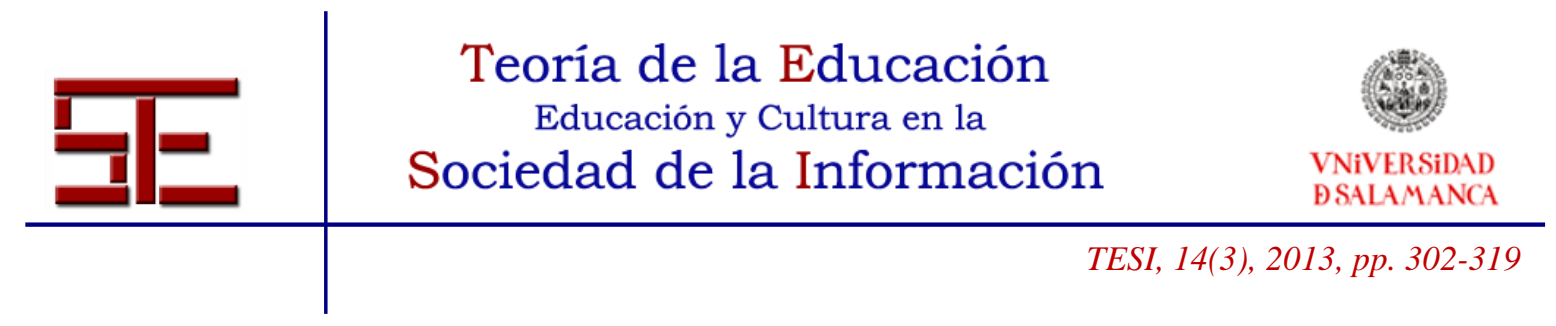

Code, J., Clarke-Midura, J., Zap, N. y Dede, C. (2011). Virtual performance assessment in immersive virtual environments. In Interactivity in e-learning: Case studies and frameworks (pp. 230-252). IGI Global.

Comisión Europea. (2007). Competencias clave para el aprendizaje permanente. Un marco de referencia europeo.

Comisión Europea. (2012). Un nuevo concepto de educación: Invertir en las competencias para lograr mejores resultados socioeconómicos. Estrasburgo: Comunicación de la Comisión al Parlamento Europeo, al Consejo, al Comité Económico y Social Europeo y al Comité de las Regiones, COM(2012) 669 final

De la Orden Hoz, A. (2011). Reflexiones en torno a las competencias como objeto de evaluación en el ámbito educativo. Revista Electrónica De Investigación Educati$v a, 13(2)$.

Delors, J. (1997). La educación encierra un tesoro. Informe a la UNESCO de la comisión internacional sobre la educación para el siglo XXI. Santillana. Ediciones UNESCO.

Erstad, O. (2010). Educating the digital generation. Exploring media literacy for the 21 st century.

Esteve, F. (2009). Bolonia y las TIC: De la docencia 1.0 al aprendizaje 2.0. La Cuestión Universitaria, 5, 59-68.

Esteve, F., Adell, J. y Gisbert, M. (2013). El laberinto de las competencias clave y sus implicaciones en la educación del siglo XXI. En II Congreso Internacional Multidisciplinar de Investigación Educativa, Tarragona.

Fundación Telefónica (2009). La generación interactiva en España. Niños y adolescentes ante las pantallas. Madrid: Ariel.

Fundación Telefónica (2011). La sociedad de la información en España 2010. Madrid: Ariel.

Gil, J. (2007). La evaluación de competencias laborales. Revista Educación XXI, 10, 83106.

Gisbert, M., Espuny, C. y González, J. (2011). INCOTIC. Una herramienta para la @ utoevaluación diagnóstica de la competencia digital en la universidad. Profesorado. Revista De Currículum Y Formación Del Profesorado, 15(1).

Hobbs, R. (2010). Digital and media literacy: A plan of action. White Paper Issued by the Aspen Institute.

ISTE. (2008). National educational technology standards for teachers. Washington DC: International Society for Technology in Education.

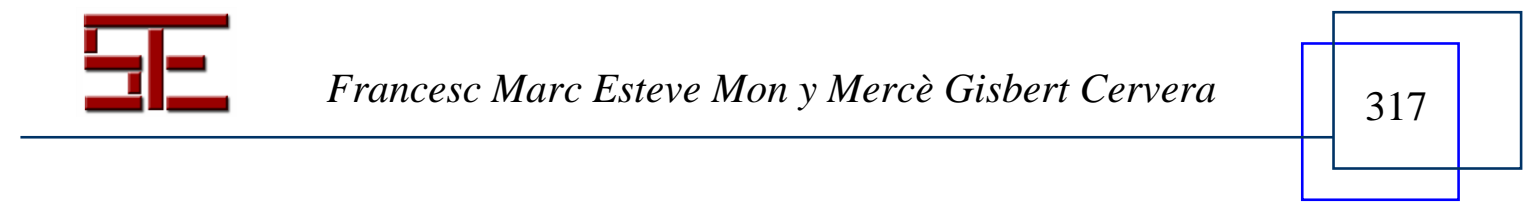




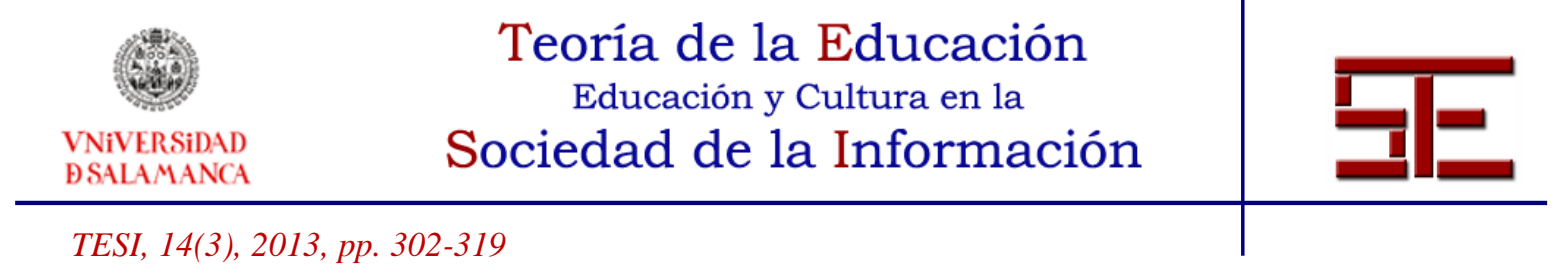

Johnson, L., Adams, S. y Cummins, M. (2012). The NMC horizon report: 2012 higher education edition. Austin, Texas: The New Media Consortium.

Livingstone, S. (2004). Media literacy and the challenge of new information and communication technologies. The Communication Review, 7(1), 3-14.

Miller, G. E. (1990). The assessment of clinical skills/competence/performance. Academic Medicine : Journal of the Association of American Medical Colleges, 65(9).

OECD. (2005). The definition and selection of key competencies (DeSeCo). Executive summary. Organisation for Economic Co-operation and Development.

Oblinger, D. G. y Oblinger, J. L. (2005). Educating the net generation. Educause.

Partnership for 21st Century Skills. (2009). Framework for 21st century learning. Tucson, Arizona: Autor.

Prades, A. (2005). Les competències transverals i la formació universitària. Thesis, Universitat de Barcelona.

Pérez Gómez, A. I. (2007). La naturaleza de las competencias básicas y sus aplicaciones pedagógicas. Consejería de Educación. Gobierno de Cantabria. Cuadernos de Educación de Cantabria.

Prensky (2001). Digital natives, digital immigrants. On the Horizon, 9(5).

Raposo, M., y Martínez, E. (2011). La rúbrica en la enseñanza universitaria: Un recurso para la tutoría de grupos de estudiantes. Formación Universitaria, 4(4), 19-28.

Redecker, C. (2013). The use of ICT for the assessment of key competences. Sevilla: Joint Research Centre, Institute for Prospective Technological Studies. European Commission.

Rice, K. (2009). Priorities in K-12 distance education: a delphi study examining multiple per- spectives on policy, practice, and research. Journal of Educational Technology \& Society, 12, 3, 163-177.

Salganik, L. H., Rychen, D. S., Moser, U. y Konstant, J. W. (1999). Proyectos sobre competencias en el contexto de la OCDE. Análisis de base teórica y conceptual. Neuchâtel, Switzerland: Organización para la Cooperación y el Desarrollo Económico (OCDE).

Salinas, D. (2002). ¡Mañana examen! La evaluación: Entre la teoría y la realidad. Grao.

Tapscott (1998). Growing up digital: The rise of the net generation. New York: McGraw-Hill.

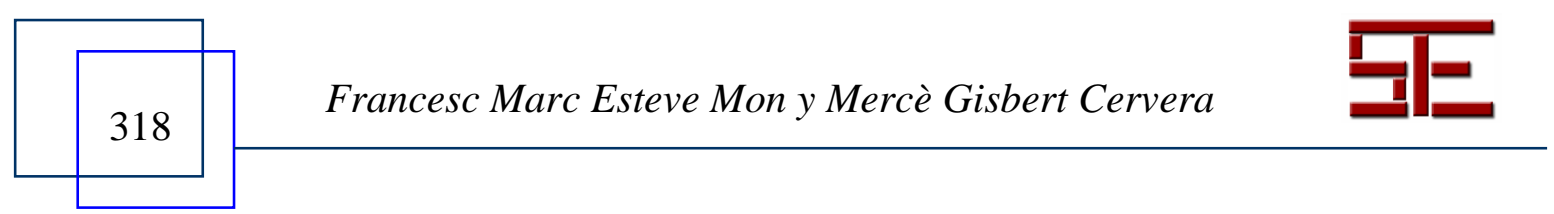




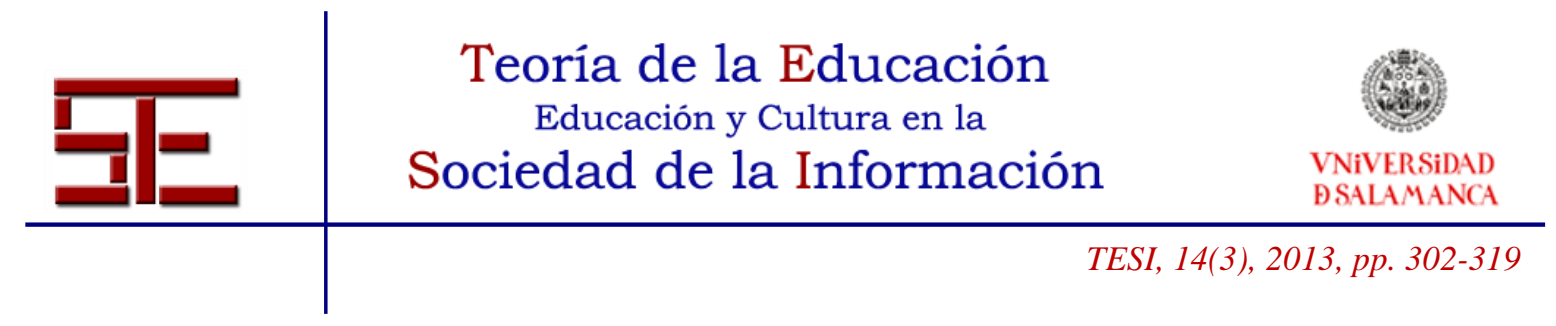

Tapscott, D. y Williams, A. D. (2009). Wikinomics: How mass collaboration changes everything. Penguin. com.

Johnson, L., Adams, S. y Cummins, M. (2012). The NMC horizon report: 2012 higher education edition. Austin, Texas: The New Media Consortium.

Unión Europea. (2006). Recomendación del parlamento europeo y del consejo de 18 de diciembre de 2006 sobre las competencias clave para el aprendizaje permanente (2006/962/CE). Diario Oficial de la Unión Europea.

Vosinakis, S., Koutsabasis, P., Zaharias, P. y Belk, M. (2011). Problem-based learning in virtual worlds: A case study in user interface design. In Proceedings of the 1st global conference: Experiential learning in virtual worlds.

Para citar el presente artículo puede utilizar la siguiente referencia:

Esteve Mon, F. M. y Gisbert Cervera, M. (2013). Explorando el potencial educativo de los entornos vir-tuales 3d. Revista Teoría de la Educación: Educación y Cultura en la Sociedad de la Información. 14(3), 302-319 [Fecha de consulta: dd/mm/aaaa]. http://campus.usal.es/ revistas_trabajo/index.php/revistatesi/article/view/11362/11778 